\title{
Intravenous infusion umbilical cord-derived mesenchymal stem cell in primary immune thrombocytopenia: A two-year follow-up
}

\author{
XIAOHUA WANG ${ }^{1,2}$, XIAOGUANG YIN ${ }^{2}$, WEI SUN $^{1}$, JIN BAI $^{1}$, YAWEN SHEN $^{1}$, \\ QIANG AO ${ }^{3}$, YONGQUAN GU ${ }^{4}$ and YING LIU ${ }^{2,5}$ \\ ${ }^{1}$ Department of Hematology, Siping Hospital of China Medical University; ${ }^{2}$ Jilin Tuhua Bioengineering Company Ltd., \\ Siping, Jilin 136000; ${ }^{3}$ Department of Tissue Engineering, China Medical University, Shenyang, Liaoning 110122; \\ ${ }^{4}$ Department of Vascular Surgery, Xuanwu Hospital of Capital Medical University, Beijing 100053; \\ ${ }^{5}$ Key Laboratory of Tissue Engineering, Siping, Jilin 136000, P.R. China
}

Received March 18, 2016; Accepted February 24, 2017

DOI: $10.3892 /$ etm.2017.4229

\begin{abstract}
Four patients with chronic refractory immune thrombocytopenic purpura (ITP) received human umbilical cord-derived mesenchymal stem cells (hUC-MSCs). The hUC-MSC dose was $5 \times 10^{7}$ to $1 \times 10^{8}$. Complete remission (CR) was achieved in three patients in 12 months and one patient in 24 months. Three patients received the second hUC-MSC transplantation with the same dose. The median time between hUC-MSC transplantation and response was 12.5 days (range, 7-16). There were no severe adverse events during and post hUC-MSC transplantation. During follow-up (median, 17 months; range, 13-24) no other immunosuppressive drugs were used post-first hUC-MSCs transplantation. In conclusion, hUC-MSC transplantation is a reasonable salvage treatment in chronic refractory ITP. Prospective randomized large-scale clinical trials are needed to further elucidate the efficacy of hUC-MSCs transplantation therapy on ITP.
\end{abstract}

\section{Introduction}

Chronic immune thrombocytopenic purpura (ITP) is an autoimmune disorder characterized by an elevated rate of platelet destruction and persistent thrombocytopenia $(1,2)$. Patients with chronic refractory ITP have the highest risk of death and disease-related or therapy-related complications $(3,4)$. Despite

Correspondence to: Professor Ying Liu, Key Laboratory of Tissue Engineering, 89 Nanyingbin Road, Siping, Jilin 136000, P.R. China E-mail: 1y3641829@163.com

Dr Yongquan Gu, Department of Vascular Surgery, Xuanwu Hospital of Capital Medical University, 45 Changchun Street, Beijing 100053 , P.R. China

E-mail: yongquan_gu1@163.com

Key words: chronic immune thrombocytopenic purpura, human umbilical cord-derived mesenchymal stem cells, intravenous infusion, safety, efficacy intense research efforts and large multicenter clinical trials, the optimal treatment for patients with chronic ITP in clinical practice remains to be determined (5).

The number of studies focusing on the therapeutic potentials of mesenchymal stem cells (MSCs) in experimental models and clinic trials are growing. One of the reasons for this growing interest can be explained by the fact that MSCs are assumed to be effective biological tools to treat degenerative diseases. In previous studies, we grafted MSCs derived from human umbilical cord-derived MSCs (hUC-MSCs) to treat non-union in rats and humans (6-8). Our results demonstrated the safety as well as the efficiency of osteoblastic differentiation of hUC-MSCs. In the present study, we describe our experience using hUC-MSCs to treat patients with chronic refractory ITP.

\section{Materials and methods}

Basic principles and ethical considerations. The protocol of the present study was approved by the Institutional Review Board and the Ethics Committee of Siping Hospital of China Medical University (Beijing, China). The trial was conducted in compliance with current Good Clinical Practice standards and in accordance with the principles set forth under the Declaration of Helsinki in 1989.

Confirmation of isolation and propagation of hUC-MSC. hUC-MSCs used in this trial were derived from two donated umbilical cords (UC) obtained from healthy mothers during routine term elective caesarean section births. Fully informed consent was obtained several weeks prior to delivery. hUC-MSCs were isolated and propagated, as previously described (6-8).

Patients. ITP was diagnosed in accordance with standard criteria and other causes of thrombocytopenia were excluded. Three adult patients with ITP having a platelet count $<30 \times 10^{9} / 1$ that persisted for at least 3 months with an inadequate or transient response to multiple therapies were treated with hUC-MSCs. The patients were willing to sign an informed consent form where they agreed to be treated in the Siping 
Table I. Patient characteristics before and post hUC-MSC transplantation.

\begin{tabular}{|c|c|c|c|c|}
\hline Characteristics & Patient 1 & Patient 2 & Patient 3 & Patient 4 \\
\hline Age (years) & 26 & 49 & 54 & 50 \\
\hline Gender & M & $\mathrm{F}$ & $\mathrm{F}$ & $\mathrm{F}$ \\
\hline Duration of disease (months) & 43 & 71 & 62 & 120 \\
\hline Previous treatments & P, V, C, IVIg, S & P, V, C, IVIg & P, IVIg, C, De, S & P, IVIg, V \\
\hline HUC-MSC transplantation times & 1 & 2 & 2 & 2 \\
\hline \multicolumn{5}{|l|}{ Platelet counts $\left(\times 10^{9} / 1\right)^{\mathrm{a}}$} \\
\hline Before therapy & 8 & 9 & 5 & 3 \\
\hline After therapy ${ }^{\mathrm{a}}$ (2 weeks) & 56 & 94 & 103 & 56 \\
\hline After therapy ${ }^{\mathrm{a}}$ (3 months) & 80 & 96 & 105 & 59 \\
\hline After therapy ${ }^{\mathrm{a}}$ (6 months) & 82 & 101 & 118 & 61 \\
\hline After therapy $^{\mathrm{a}}$ (12 months) & 189 & 84 & 234 & 116 \\
\hline After therapy ${ }^{\mathrm{a}}$ (24 months) & 134 & & & \\
\hline \multicolumn{5}{|l|}{ Bleeding $^{\mathrm{b}}$} \\
\hline Before therapy & $\begin{array}{l}\text { Skin, genitourinary } \\
\text { bleeding }\end{array}$ & Skin & Epistaxis & $\begin{array}{l}\text { Skin, genitourinary } \\
\text { bleeding }\end{array}$ \\
\hline After therapy & No & No & Mucosal & Skin \\
\hline Time to response (days) & 7 & 13 & 16 & 14 \\
\hline Time to maximum response (days) & 31 & 53 & 42 & 58 \\
\hline Overall response & Yes & Yes & Yes & Yes \\
\hline Response duration (months) ${ }^{c}$ & Yes, 24 & Yes, 18 & Yes, 13 & Yes, 13 \\
\hline
\end{tabular}

${ }^{a}$ Platelet count measurements were carried out at the end of the following time points post hUC-MSC transplantation. ${ }^{\mathrm{b}} \mathrm{Major}$ skin indicates diffuse ecchymosis; mucosal, intrabuccal hemorrhagic vesicles, or prolonged epistaxis; and intestinal and menorrhagia, gastrointestinal, and

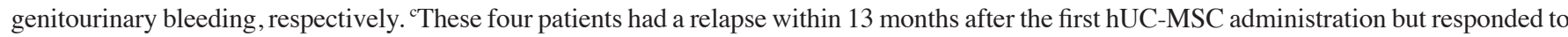
the second hUC-MSC treatment, and they all sustained response for $>8$ months. hUC-MSCs, human umbilical cord-derived mesenchymal stem cells; M, male; F, female; P, prednisone; V, vincristine; C, cyclosporin; IVIg, intravenous immunoglobulins; S, splenectomy; De, dexamethasone.

Hospital of China Medical University. The general characteristics of the patients are presented in Table I.

Intravenous infusion of $h U C-M S C s$. hUC-MSCs $(10 \mathrm{ml})$ with a cell density of $5 \times 10^{6}$ to $1 \times 10^{7} / \mathrm{ml}$ was given intravenously at a rate up to $12.5 \times 10^{6} / \mathrm{min}$ and flushed with $20 \mathrm{ml}$ saline to ensure full cell dose delivery. Once the needle was fully withdrawn, the puncture site was wrapped with sterile dressing. Patients remained in the supine decubitus on the operation bed for another $30 \mathrm{~min}$ before off-bed activities and antibiotics were given to prevent infection. Patients' conditions were monitored (temperature, blood pressure, pulse and oxygen saturation) at 15, 30, 45 and $60 \mathrm{~min}$, and then once every hour for a minimum of $4 \mathrm{~h}$.

Measurement of patelet related parameters. Platelet-related parameters were analyzed before the operation and at several time points post-transplantation using an automated blood cell counter model LH-750 (Beckman Coulter, Inc., Brea, CA, USA).

Clinical and functional assessment. i) Primary safety assessments included monitoring and recording of all the adverse events as well as the serious adverse events. The patients were monitored (temperature, blood pressure, pulse and oxygen saturation) at 15,30,45 and $60 \mathrm{~min}$, and then once every hour for a minimum of $4 \mathrm{~h}$. They were discharged $24 \mathrm{~h}$ post-transplantation if they were not febrile and hemodynamically stable, with no signs of infection or any type of allergic reaction. Any abnormal reactions within 3 months were considered to be linked to transplantation.

ii) As exploratory secondary end-points we investigated the efficacy of hUC-MSC infusion as assessed by platelet-related parameters, at baseline and at a series of time-points (2 weeks and 3, 6, 12 and 24 months post-first hUC-MSC administration). Initial response evaluation was made at the end of the second week after treatment initiation. Complete remission (CR) was considered when the platelet count was $>1 \times 10^{11} / 1$, partial remission $(\mathrm{PR})$ if platelets were $>5 \times 10^{10} / 1$, and minimal response $(\mathrm{MR})$ if the platelet count was between $3 \times 10^{10} / 1$ and $5 \times 10^{10} / 1$. No response was platelet count that remained unchanged. Response was classified as sustained (SR) when it was stable for a minimum of 6 months. Relapse was defined as a decline in platelet count to $<30 \times 10^{9} / 1$ and/or the need for ITP rescue treatments.

Pharmacological therapy protocol. Pharmacological therapy consisted of: i) inhaling high doses of steroids and prednisone (1 mg/kg, p.o., once daily); ii) vincristine (2 mg, once per month, i.v.); iii) intravenous immunoglobulins ( $\gamma$ globulin), $0.4 \mathrm{~g} / \mathrm{kg}$, once daily, i.p.; and iv) cyclosporine ( $3 \mathrm{mg} / \mathrm{kg}$, p.o., once daily). 

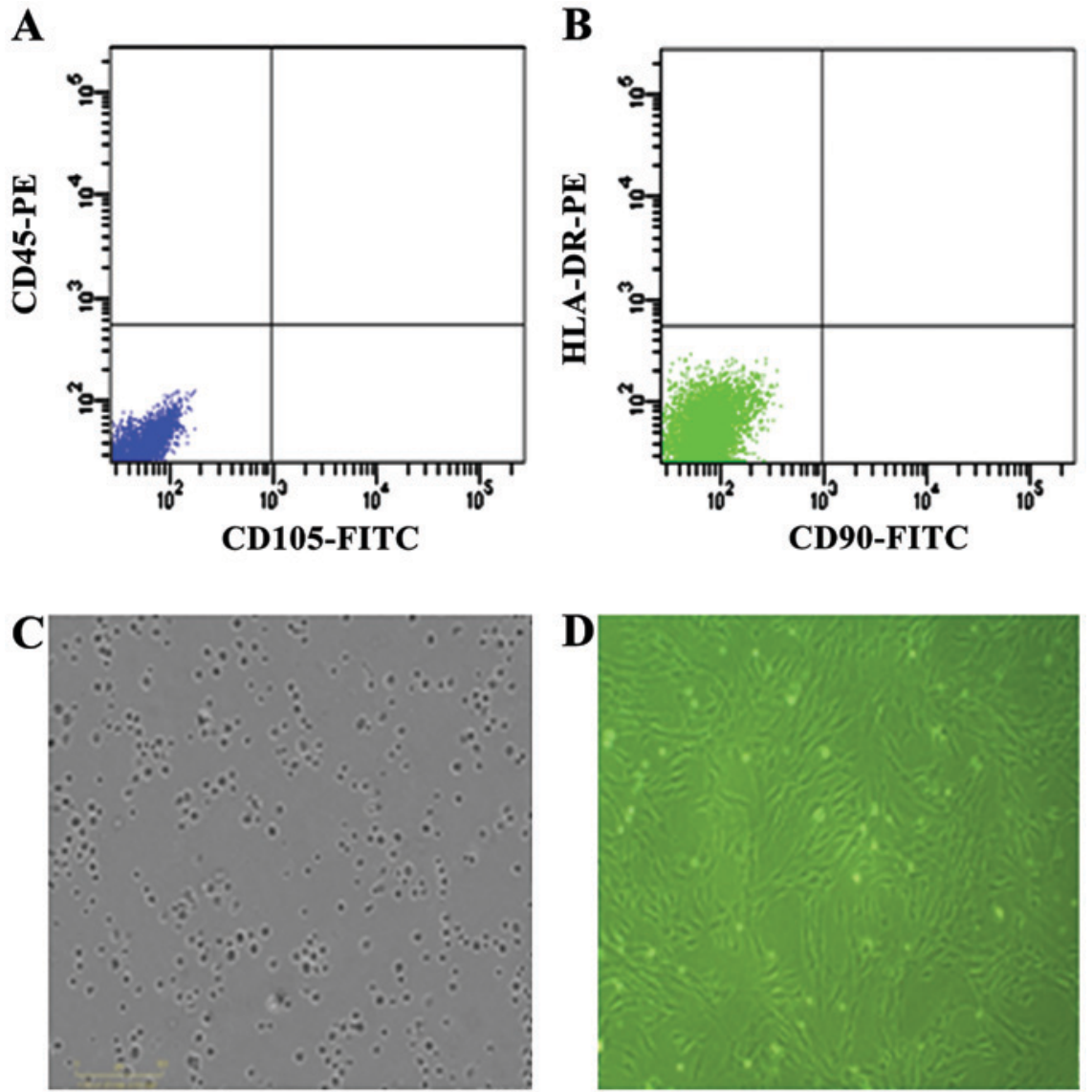

Figure 1. Evaluation of hUC-MSCs. (A) The cells derived from UC were observed at $24 \mathrm{~h}$ after they were seeded; (B) three days after inoculation; (C) hUC-MSC surface markers: CD105 and CD45, (D) hUC-MSC surface markers, HLA-DR and CD90. hUC-MSCs, human umbilical cord-derived mesenchymal stem cells; UC, umbilical cord.

Statistical analysis. Statistical analyses were performed using SPSS 16.0 software (Chicago, IL, USA). Safety and exploratory efficacy secondary endpoints were observed for each patient against the baseline values. $\mathrm{P}<0.05$ was considered statistically significant.

\section{Results}

Evaluation of hUC-MSCs. Cells derived from UC were observed $24 \mathrm{~h}$ after they were seeded (Fig. 1A), during the time that part of the round mononuclear cells was adherent. Three days after inoculation, small colonies of the adherent cells with typical fibroblast-shaped morphology were obtained (Fig. 1B). These primary cells reached monolayer confluence, after planting for 5-6 days, when they were passaged for the first time. Fifth passage cells were analyzed by flow cytometry and were strongly positive for CD105 and CD90, but negative for CD45 and HLA-DR (Fig. 1C and D).

Patient characteristics. Clinical characteristics of patients who participated in the present study are summarized in Table I. The patients were 3 females and 1 male, with an age range of 26-54 years (median, 44.75 years). Median duration of ITP before hUC-MSC transplantation was 74 months (range, 13-120 months) and the median number of prior treatments was 2 months (range, 1-3 months), which included splenectomy, prednisone, intravenous immune globulin, cyclosporine and vincristine. All the patients had a history of major bleeding and those episodes were often transient but recurrent. Major hemorrhagic events included genitourinary bleeding, diffuse ecchymosis and prolonged epistaxis.

Clinical therapeutic effect of hUC-MSCs. Results of hUC-MSC treatment are shown in Table I. Overall responses were reached in all the patients at the end of the second week after the hUC-MSCs had been administered. The patients achieved a platelet count of $>50 \times 10^{9} / 1$ and 2 patients achieved a platelet count of $>90 \times 10^{9} / 1$. The median platelet count on treatment was $77.25 \times 10^{9} / 1$ (range, $56 \times 10^{9}$ to $10.3 \times 10^{10} / 1$ ). The median time to response and the median time to maximum response were 12.5 days (range, 7-16 days) and 46 days (range, 31-53 days), respectively. One patient sustained response after a single course of hUC-MSCs without any further therapy during the follow-up. Major bleeding episodes did not occur. The remaining 3 patients (patients 2-4) had a relapse within 12 months after the first hUC-MSC administration but responded to the second hUC-MSC treatment. The time to the second response for patients 2,3 and 4, was 13 , 16 and 18 days, respectively, whereas the time to the second maximum response was 34,38 and 43 days, respectively. All the patients achieved a sustained response of $>10$ months.

Safety outcomes. No serious or clinically significant side effects were observed during the entire study period. During 
the whole follow-up period, neither ectopic tissue formation nor other illnesses related to the hUC-MSCs treatment were recorded in the patients.

\section{Discussion}

In the present study, we evaluated the response rate achieved in 4 patients with chronic and refractory ITP after hUC-MSC intravenous infusion in 24 months. The clinical median relieved time of symptoms after one transplant was 17 months. To the best of our knowledge, this is the first study on the efficiency of hUC-MSC transplantation treatment for ITP patients. Our experimental results supported cell therapy for ITP.

The hUC-MSCs used in the present study met the criteria of the International Society for Cell Therapy (9-11). In this experiment, we observed three favorable responses (100\%) in 12 months after one hUC-MSC transplantation. Patient 1 recurrence occurred in 29 months, while the other three patients had a recurrence in almost 13 months. Our data suggested that symptom alleviation was not complete. Based on the characteristics of the evolution of our group of patients, we may emphasize a few important points: i) hUC-MSC transplantation is beneficial for the recovery of bone marrow megakaryocytes, in order that patients can achieve long-term relief; and ii) some cytokines are reduced in this reaction, and the effect of hUC-MSC transplantation is decreased. Thus, the underlying mechanisms of hUC-MSC therapy on ITP need further exploration.

In addition, the pathophysiology of ITP is complex and abnormalities of the B- and T-cell compartments have been identified. Furthermore, MSCs in ITP patients have a reduced proliferative capacity and a lower inhibitory effect on activated T-cell proliferation compared with MSC from healthy donors (12). These abnormalities indicate a possible role for MSC malfunction in the physiopathology of the disease and may have therapeutic implications. In the present study, we did not monitor changes in B-cell counts and platelet autoantibodies, which may influence the response as observed in other trials $(13,14)$, therefore we cannot confirm the effect of hUC-MSC transplantation on the immune regulation. Future large-scale trials are likely to be designed to investigate the immunomodulatory characteristics of hUC-MSCs on treating ITP.

In conclusion, hUC-MSC transplantation is safe and effective for treating ITP. Our data suggest that MSC therapy may be a reasonable salvage treatment in severe, potentially life-threatening, refractory ITP. The optimal period of hUC-MSC transplantation for treating ITP is once a year. Prospective randomized clinical trials are needed to elucidate the efficacy of hUC-MSC transplantation therapy on ITP in the future.

\section{Acknowledgements}

The present study has been supported by the National High Technology Research and Development Program, 863 Program, nos. 2011AA020101 and 2012A020905.

\section{References}

1. Berchtold P and McMillan R: Therapy of chronic idiopathic thrombocytopenic purpura in adults. Blood 74: 2309-2317, 1989.

2. Imbach P: Treatment of immune thrombocytopenia with intravenous immunoglobulin and insights for other diseases. A historical review. Swiss Med Wkly 142: w13593, 2012.

3. McMillan R and Durette C: Long-term outcomes in adults with chronic ITP after splenectomy failure. Blood 104: 956-960, 2004.

4. Portielje JE, Westendorp RG, Kluin-Nelemans HC and Brand A: Morbidity and mortality in adults with idiopathic thrombocytopenic purpura. Blood 97: 2549-2554, 2001.

5. Saeidi S, Jaseb K, Asnafi AA, Rahim F, Pourmotahari F, Mardaniyan S, Yousefi H, Alghasi A, Shahjahani M and Saki N: Immune thrombocytopenic purpura in children and adults: a comparative retrospective study in IRAN. Int J Hematol Oncol Stem Cell Res 8: 30-36, 2014.

6. Qu Z, Guo S, Fang G, Cui Z and Liu Y: AKT pathway affects bone regeneration in nonunion treated with umbilical cord-derived mesenchymal stem cells. Cell Biochem Biophys 71: 1543-1551, 2015.

7. Qu Z, Guo L, Fang G, Cui Z, Guo S and Liu Y: Biological characteristics and effect of human umbilical cord mesenchymal stem cells (hUC-MSCs) grafting with blood plasma on bone regeneration in rats. Cell Biochem Biophys 63: 171-181, 2012.

8. Qu Z, Fang G, Cui Z and Liu Y: Cell therapy for bone nonunion: a retrospective study. Minerva Med 106: 315-321, 2015.

9. Fang B, Mai L, Li N and Song Y: Favorable response of chronic refractory immune thrombocytopenic purpura to mesenchymal stem cells. Stem Cells Dev 21: 497-502, 2012.

10. Rodeghiero F, Stasi R, Gernsheimer T, Michel M, Provan D, Arnold DM, Bussel JB, Cines DB, Chong BH, Cooper N, et al: Standardization of terminology, definitions and outcome criteria in immune thrombocytopenic purpura of adults and children: report from an international working group. Blood 113: 2386-2393, 2009.

11. Dominici M, Le Blanc K, Mueller I, Slaper-Cortenbach I, Marini F, Krause D, Deans R, Keating A, Prockop Dj and Horwitz E: Minimal criteria for defining multipotent mesenchymal stromal cells. The International Society for Cellular Therapy position statement. Cytotherapy 8: 315-317, 2006.

12. Pérez-Simón JA, Tabera S, Sarasquete ME, Díez-Campelo M, Canchado J, Sánchez-Abarca LI, Blanco B, Alberca I, Herrero-Sánchez C, Cañizo C, et al: Mesenchymal stem cells are functionally abnormal in patients with immune thrombocytopenic purpura. Cytotherapy 11: 698-705, 2009.

13. Li X, Zhong H, Bao W, Boulad N, Evangelista J, Haider MA Bussel $\mathrm{J}$ and Yazdanbakhsh K: Defective regulatory B-cell compartment in patients with immune thrombocytopenia. Blood 120: 3318-3325, 2012.

14. Cines DB, Bussel JB, Liebman HA and Luning Prak ET: The ITP syndrome: pathogenic and clinical diversity. Blood 113: 6511-6521, 2009. 